\title{
Coherent qubits stability and quantum phase transitions in the Lipkin-Meshkov-Glick model
}

\author{
Enrique López-Moreno · M. Grether
}

Received: 6 June 2014 / Accepted: 12 August 2014 / Published online: 27 August 2014

(C) Chapman University 2014

\begin{abstract}
The understanding and manipulation of information at quantum level requires an adequate treatment of phenomena such as qubits stability, singular behavior of Fidelity evolution at the event of quantum phase transitions, tunneling, and parametric dependence of physical observables, such as the magnetization, under control variables manipulation. In the present work we consider the Lipkin-Meshkov-Glick model Hamiltonian, useful in the treatment of a kind of single-molecule magnets. Phase transition behavior is treated by means of catastrophe theory. We show that quantum and semiclassical behavior are completely organized by the separatrix of the system. Catastrophe theory and the coherent states formalism represent powerful tools in the analytical treatment of quantum information and its semiclassical behavior. Coherent states of the model demonstrate their importance at the semiclassic critical points. In particular, these states represent very closely the ground state of the system. Coherent qubit states are introduced by means of the coherent state representation of the system states. Singular character of Fidelity, due to tunneling at a phase transition point, can be numerically adjusted very closely to a Lorentzian curve.
\end{abstract}

Keywords Quantum phase transitions - Algebraic models - Quantum state engineering and measurements · Quantum information and quantum mechanics

\section{Introduction}

Quantum coherence is a central concept in physics, and applications in quantum information processing depend on its understanding and manipulation. Arrays of interacting spins have attracted much attention in recent years [1]. Entanglement of quantum states and phase transitions can be studied in these simple systems. Entanglement represents an estimator for detecting quantum phase transitions, representing a way to detect quantum phase transitions in anisoropic $S=1 / 2$ antiferromagnetic chains [2-4]. Single-molecule magnets (SMM) behave like nanoscale magnets [5], with quantum behavior at low temperatures [6-8]. In crystalline $\mathrm{Fe}_{8}$ molecular magnets, the electronic spins are structurally ordered, and quantum coherence is observed in collective magnon motion of the spins, rather

E. López-Moreno $(\varangle) \cdot$ M. Grether

Departamento de Física, Facultad de Ciencias, Universidad Nacional Autónoma de México, 04510 Mexico, DF, Mexico e-mail: elmlvi@gmail.com

M. Grether

e-mail: mdgg@ciencias.unam.mx 
than in single qubit dynamics $[9,10]$. Likewise, it has also been considered that quantum phase transitions (QPT) at zero temperature are fundamental phenomena [11] extensively studied for many-body systems in their ground state and recently between states through their excitation spectra [12]. In the studies in the nuclear shape problem [13], phase transitions in quantum systems have gained an expanding interest. At the semiclassical approximation, this singular behavior has been observed in the minima of the corresponding energy surface (ES) associated with the physical system. ES is evaluated as the expectation value of the Hamiltonian in terms of the coherent state formalism. These singularities are found for some critical values of the system parameters as they continuously vary, thus giving the loci of the separatrix on the parameter space.

Lipkin-Meshkov-Glick Hamiltonian (LMG) [14] can represent a two-level system of identical particles interacting through particle-particle and particle-hole channels, where phase transitions from single particle to collective motion can be visualized [15,16]. A characterization of phase transitions and related accidental degeneracies associated with the LMG model Hamiltonian by means of its associated separatrix was presented in [1], and quantum energy degeneration of the LMG model Hamiltonian was presented in analytical form as hyperbolae indicating the regions in the Hamiltonian parameter space. A detailed analysis of the behavior of the classical trajectories and quantum properties in the vicinity of the separatirx were studied in [17]. Also, in this last reference, it was shown that the global behavior of the quantum-level structure and their corresponding wave function are described appropriately by means of this separatix. The evolution of the energy surfaces as a function of the control parameters and its relation with the exact quantum states were left as a future work, and part of the present paper presents an approach to this problem. LMG has also been useful in many investigations involving the relation between the classical and quantum properties in many-body systems, quantum phase transition problems, [18-20], and in various aspects of quantum information, such as Fidelity [21], that mathematically is represented by the overlap between two ground states corresponding to a slight difference in their driving parameter. Under a QPT, the ground state of a system undergoes a structural change as a control parameter crosses the corresponding critical point, and Fidelity depends on a small change of the control parameter, that is, quantum criticality promotes a decay of Fidelity [22].

In the present work we are interested in LMG algebraic model as it was used in the past to study SMM; however, the methods developed here are applicable for general values of the system parameters and other systems in which LMG has been very useful [1,17]. Here, as a matter of convenience, we limit ourselves to those parameters related to the magnetic properties of the $J=10, \mathrm{Fe}_{8} \mathrm{SMM}$, well described by the spin Hamiltonian Eq. (1), and we adopted the parameter values related to those given in [5]. Quantum energy spectrum structure and the corresponding semi-classical ES are intimately related to each other by means of the phase transitions associated with all its critical points. Phase transition behavior, and its character, shall be treated by means of catastrophe theory. General quantum and semiclassical behavior are completely organized by means of the separatrix of the system. We show the combined action of catastrophe theory and coherent state formalisms as a powerful tool in the study of relevant aspects in quantum information, as it is the Fidelity.

\section{Model spin Hamiltonian}

LMG Hamiltonian [14] has been successful when treating $\mathrm{Fe}_{8}$ molecular magnets:

$$
\widehat{H}=\frac{\epsilon}{4 J} \hat{J}_{z}+\frac{\gamma}{2 J(2 J-1)} \hat{J}_{z}^{2}+\frac{\lambda}{4 J(2 J-1)}\left(\hat{J}_{+}^{2}+\hat{J}_{-}^{2}\right)
$$

where $\hat{J}_{z}, \hat{J}_{ \pm}$denote the spherical components of the angular momentum operator $\hat{J}$; here, $J$ is the highest $\hat{J}_{z}$ eigenvalue, and $\epsilon, \gamma$, and $\lambda$ are real numbers representing the interaction strength control parameters. The total energy would grow faster than linearly with $J$. In order to solve this question, we write an intensive quantity for the Hamiltonian by means of the, $1 / J$, one-body factors and, $1 /(J(2 J-1))$, two-body factors. Let the set, $\left\{\left|\Gamma_{k}\right\rangle\right\}$, denote a complete eigenvector states basis for $\widehat{H}$ : 
$\widehat{H}\left|\Gamma_{k}\right\rangle=\Lambda_{k}\left|\Gamma_{k}\right\rangle, \quad 1 \leq k \leq 2 J+1$,

where $\left\{\Lambda_{k}\right\}$ are the corresponding energy eigenvalues.

\section{Angular-momentum basis}

The eigenstates of the operator $\hat{J}_{z}$ are not in general eigenstates the system described by the Hamiltonian (1). This set, however, also constitutes a complete set of functions and contains $(2 J+1)$ basis vectors, defined as usual: $\hat{J}_{z}|J, m\rangle=m|J, m\rangle, \quad-J \leq m \leq J$. Normalized states can be obtained by applying the shift-up operator to the $\hat{J}_{z}$ operator's ground state: $|J,-J\rangle,(J+m)$-times, as follows:

$|J, m\rangle=\left(\begin{array}{c}2 J \\ J \pm m\end{array}\right)^{-1 / 2} \frac{\hat{J}_{+}^{(J+m)}}{(J+m) !}|J,-J\rangle$.

These are the Dicke states [23]. In this paper, numerical work shall be realized within Dicke basis. Even though the eigenstates of the system do not have a definite angular momentum, $\hat{J}_{z}$, any one of these has a probability, $\left|c_{m}^{(k)}(\epsilon)\right|^{2}$, of being observed with this value, where

$\left|\Gamma_{k}(\epsilon)\right\rangle=\sum_{m=-J}^{m=J} c_{m}^{(k)}(\epsilon)|J, m\rangle$

\section{Coherent states. Energy surface}

A geometrical interpretation of Hamiltonian (1) can be realized by means of a semiclassical approximation established by evaluating the energy expectation value in terms of the coherent states basis [23]: $E^{ \pm}=\left\langle\zeta^{ \pm}|\widehat{H}| \zeta \pm\right\rangle$. Spin coherent states are defined by variable $\zeta$ that represents a point in the complex plane mapped by a stereo-graphical projection of a point on a unit sphere $\Sigma_{0}$, from one of its poles. Thus, if $(x, y, z)$ are cartesian coordinates of a point over this $\Sigma_{0}$ surface, then $(\theta, \phi)$ are its polar and azimuthal angles, respectively. Then we have the corresponding coherent state, $\left|\zeta^{ \pm}(x, y)\right\rangle$ :

$\left|\zeta^{ \pm}\right\rangle=\sum_{m=-J}^{J}\left(\frac{1 \pm z}{2}\right)^{J}\left(\frac{x+i y}{1 \pm z}\right)^{J+m} \sqrt{\left(\begin{array}{c}2 J \\ J+m\end{array}\right)}|J, m\rangle$.

where $z=\sqrt{1-x^{2}-y^{2}}$. For a given set of parameter values, the Hamiltonian expectation value, $E^{ \pm}$, represents the semiclassical energy surface (ES). A direct evaluation from Eqs. (1) and (5), gives

$E^{ \pm}=\frac{\mp \epsilon}{4} \sqrt{1-x^{2}-y^{2}}-\gamma_{x} x^{2}-\gamma_{y} y^{2}+\frac{\gamma J}{2(2 J-1)}$.

Here, $\gamma_{x} \equiv \frac{\gamma-\lambda}{4}$, and, $\gamma_{y} \equiv \frac{\gamma+\lambda}{4}$. This energy $E^{ \pm}(x, y ; \epsilon)$, for a given $\epsilon$, is considered as a double sheet function of $(x, y)$ variables. The $J-, \gamma-$, and $\lambda$-values play the role of a fixed parameter set defining a given spin system, as it is the case for $\mathrm{Fe}_{8}$ molecule; $\epsilon$ is a control parameter, related to the external magnetic field along the easy axis in this example. Semiclassical equilibrium and its stability properties can be studied considering ES critical points. We shall show that relevant quantum behavior such as energy level structure and magnetization of the system are ruled in the large by ES attributes. 


\section{ES critical points. Classical phase transitions}

General behavior of the ES, Eq. (6), obtained when the control parameters $\epsilon, \gamma$, and $\lambda$, are varied, defines a whole family of functions. A complete analysis of this family of functions can be given by means of the application of catastrophe formalism, and thus we are able to determine the degeneracy of critical points, the bifurcation sets of the energy surface, and the locus of points in the space of control parameters at which a phase transition occurs from one local critical point to another. One can also get the organization of all the critical points, particularly for the equilibrium points, and their stability within the control parameters space. This method was investigated elsewhere for the special case of non-axially symmetric spin systems [24]. The separatrix, or bifurcation set is given in the present work by the loci of critical parameter points: $\epsilon_{\mathrm{c}}=0, \pm \epsilon_{x}, \pm \epsilon_{y}$; as well as by the critical energy surface (CES), that is, the $E^{ \pm}$in Eq. (6) evaluated at all the critical points; all these critical values are given in Table 1. A plot of the CES is presented in Fig. 1. In between the regions of the separatrix the behavior of the system remains qualitatively unchanged; but at this loci, sudden classical and quantum behavior changes are observed because equilibria points are either created or destroyed.

The functional form of the minima values of the ES is easily found as:

$E_{\min }= \begin{cases}+|\epsilon / 4|+\gamma J /(2(2 J-1)), & \text { if } \epsilon \leq 0 \\ -|\epsilon / 4|+\gamma J /(2(2 J-1)), & \text { if } \epsilon \geq 0\end{cases}$

Coherent state energy minimum goes through a first-order phase transition at the critical parameter $\epsilon=0$ value, as it is clear from Eq. (7). The ratio, $E_{\min } / \Lambda_{g}(\epsilon)$, of this coherent state energy minimum, to the quantum ground state energy, as a function of parameter $\epsilon$, shows a close proximity between these values, improving from $99.87 \%$ at $\epsilon=0$, to $100 \%$ in the limit $\epsilon \rightarrow \pm \infty$.

\section{Quantum vs. semiclassic phase transitions}

The energy level structure, and its relation to the corresponding semiclassical ES, is also shown in Fig. 1; there, the role of the CES and the separatrix of parameter space is presented. It is important to observe that energy level anticrossings, and staggering, are ruled by the CES regions. In this sense, CES can be considered as part of the separatrix. A CES region is observed where the Hamiltonian eigenstates have a regular behavior with a great overlap with Dicke states; that is: $\left|\Gamma_{k}(\epsilon)\right\rangle \simeq|J, k\rangle$, for any of the $k$ th, of the $(2 J+1)$ eigenstates. This regular region is defined by the corresponding eigenvalue conditions:

Table 1 Critical variables and parameters of function $E^{ \pm}$

\begin{tabular}{lll}
\hline$\left(x_{\mathrm{c}}, y_{\mathrm{c}}\right)$ critical point & Character parameter region & ES \\
\hline$(0,0)$ & Absolute minimum $0 \leq \epsilon \quad(\epsilon \leq 0)$ & $E^{+}\left(E^{-}\right)$ \\
$(0,0)$ & Relative minimum $0 \leq \epsilon \leq \epsilon_{y} \quad\left(-\epsilon_{y} \leq \epsilon \leq 0\right)$ & $E^{-}\left(E^{+}\right)$ \\
$(0,0)$ & Saddle point $\epsilon_{y} \leq \epsilon \leq \epsilon_{x} \quad\left(-\epsilon_{x} \leq \epsilon \leq-\epsilon_{y}\right)$ & $E^{-}\left(E^{+}\right)$ \\
$(0,0)$ & Absolute maximum $\epsilon_{x} \leq \epsilon \quad\left(\epsilon \leq-\epsilon_{x}\right)$ & $E^{-}\left(E^{+}\right)$ \\
$\left(0, \pm y_{\mathrm{c}}\right)$ & Saddle point $0 \leq \epsilon \leq \epsilon_{y} \quad\left(-\epsilon_{y} \leq \epsilon \leq 0\right)$ & $E^{-}\left(E^{+}\right)$ \\
$\left( \pm x_{\mathrm{c}}, 0\right)$ & Absolute maximum $0 \leq \epsilon \leq \epsilon_{x} \quad\left(-\epsilon_{x} \leq \epsilon \leq 0\right)$ & $E^{-}\left(E^{+}\right)$
\end{tabular}

The first column indicates the corresponding critical point. The second column gives its character and region in the control parameter space. Here, critical variables are $x_{\mathrm{c}} \equiv \sqrt{1-\left(\epsilon / \epsilon_{x}\right)^{2}}, y_{\mathrm{c}} \equiv \sqrt{1-\left(\epsilon / \epsilon_{y}\right)^{2}}$, and the critical parameters are $\epsilon_{x} \equiv 2|(\gamma-\lambda)|, \epsilon_{y} \equiv$ $2|(\gamma+\lambda)|$ 
Fig. 1 a Quantum energy levels $\Lambda_{n}$, and (b) CES, in the control parameter space $\epsilon$; continuous (dashed) line corresponds to $E^{+}\left(E^{-}\right)$. CES, evaluated at all its critical parameters: $\epsilon_{\mathrm{c}}=0, \pm \epsilon_{x}, \pm \epsilon_{y}$, is indicated by small circles (a)
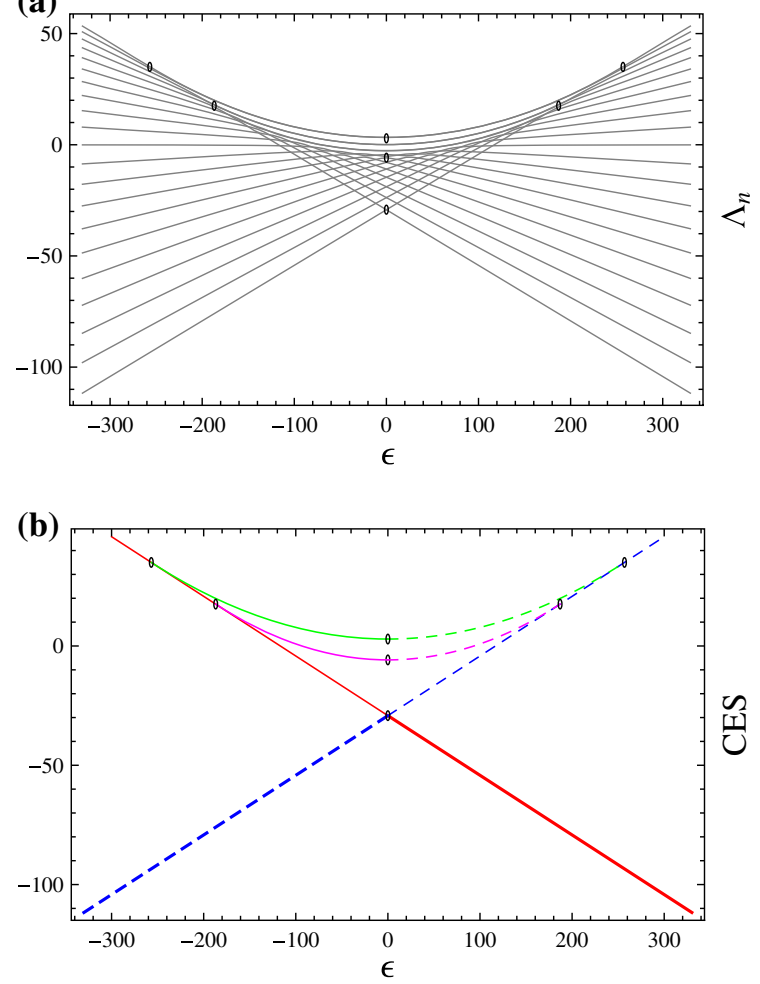

$E^{-}(0,0 ; \epsilon) \leq \Lambda_{k}(\epsilon) \leq E^{+}(0,0 ; \epsilon)$, if $\epsilon \leq 0$;

$E^{+}(0,0 ; \epsilon) \leq \Lambda_{k}(\epsilon) \leq E^{-}(0,0 ; \epsilon)$, if $\epsilon \geq 0$.

Within this region, the Hamiltonian (1) is almost diagonal in the Dicke basis states:

$\langle J, m|\widehat{H}| J, m\rangle \simeq \frac{m}{4 J} \epsilon+\frac{\gamma m^{2}}{2 J(2 J-1)}$,

That is, for a given set of parameters: $\{J, \gamma, \lambda\}$, all the energy levels constitute very closely an uniparametric family of straight lines of variable $\epsilon$, and $m$-quantum number as a parameter. Coherent energy minima $E^{ \pm}(0,0 ; \epsilon)$ (7) take the exact value for the corresponding, $m=\mp J$, into the above equation. In between the CES region limited by the $(x, y)=(0,0)$, relative minima, and the $(x, y)=\left(0, \pm y_{\mathrm{c}}\right)$, saddle points given at Table 1 , the energy level structure possess a number of avoided energy crossings, located approximately at the intersection formed from the continuation of the straight lines Eq. (9); in this way, the evolution of the system exhibits quantum phenomena in its dynamics as it is observed for molecular nano magnets, due to tunneling, at these parameter values. Quantum tunneling of magnetization can be advantageous for some potential applications of SMMs, for example, in providing the quantum superposition of states required for quantum computing [7,8]. Finally, CES also plays the role of a quantum separatrix for the region in between the saddle points last mentioned and the $(x, y)=\left( \pm x_{\mathrm{c}}, 0\right)$, absolute maxima; there, the nature of the avoided crossings is not the reticular one as before, but it is realized pairwise. 


\section{Coherent qubits}

It is very important to note that, while in the previous work cited above, [1], and [17], coherent states play the role of trial states to find a semiclassical approximation to the ground state of the system and also to the first excited state when parity is restored within this formalism; this in not, in any way, a purpose by itself in the present work. In the present manuscript, coherent states are the atomic coherent states treated by themselves with a different approach. Here, they play a more general and important role in the global approach of the problem, as we explain in the following two paragraphs:

Field coherent states have maintained a central position in the development of quantum optics since its introduction by Glauber in 1963 [25]. In a dual treatment, the atomic coherent states were introduced [23], closely related to each other, and with a number of useful properties in common. Usefulness of the field coherent states is firmly established. There also exist physically useful applications of the atomic coherent states, as in the approximate solutions of quantum optics Hamiltonians involving the description of the interaction between $\mathrm{N}$ atoms and an electromagnetic field confined to a cavity of finite volume. Also, in a second example, atomic coherent states prove to be very important in the problem of construction of thermodynamic partition functions for large class of spin Hamiltonians. Certainly, these applications depend in a crucial way on the overcompleteness of the atomic coherent states. The resemblance of field, and atomic, coherent states is not solely formal, nor does it come about because the treatments of both follow the same basic pattern; it comes for sound reasons firmly based on group theory [26]. Bloch sphere was introduced by Bloch [27] for the discussion of the nuclear induction experiment; the atomic coherent states, within the particular case of two-level atoms, are called Bloch states. Dicke and Fock states are related to the Bloch and Glauber states.

Quantum coherence is a central concept in physics. Applications such as quantum information processing depend on its understanding and manipulation. In crystalline $\mathrm{Fe}_{8}$ molecular magnets electronic spins are structurally ordered, and quantum coherence is observed in collective magnon motion of the spins, rather than in single qubit dynamics $[9,10]$. In what follows we introduce the concept of coherent qubit using the cartesian coordinates of a coherent state in Eq. (5) and the Bloch sphere.

A qubit [28], $|\chi\rangle=a|0\rangle+b|1\rangle$, where $|a|^{2}+|b|^{2}=1$, is represented as a given point over a unitary spherical surface, $\Sigma_{0}$, by means of the spherical coordinates, $(\theta, \phi)$, the Bloch sphere:

$a=\cos (\theta / 2), \quad b=\mathrm{e}^{i \phi} \sin (\theta / 2)$.

This point maps the qubit, $|\chi\rangle$, into a cartesian Bloch vector: $(x, y, z)=(\sin \theta \cos \phi, \sin \theta \sin \phi, \cos \theta)$. Let the coordinates of the coherent state, $\left|\zeta^{ \pm}(x, y)\right\rangle$, in Eq. (5), be treated as the cartesian coordinates of a Bloch vector. In this way, we introduce these states as a definition of the coherent qubit states (CQS) basis. Any quantum state of Hamiltonian (1) can be expanded into its associated coherent states basis, and then CQS let us handle quantum information for the most general state evolution of the system. Coherent states, Eq. (5), in terms of qubits amplitudes, $\left|\zeta^{ \pm}(a, b)\right\rangle$, are

$\left|\zeta^{\{ \pm\}}\right\rangle=\sum_{m=-J}^{J}\left(\begin{array}{c}2 J \\ J+m\end{array}\right)^{\frac{1}{2}}(a b)^{J}\left\{\begin{array}{c}(b / a)^{m} \\ \left(a / b^{*}\right)^{m}\end{array}\right\}|J, m\rangle$.

\section{Qubits stability}

Let the system be prepared at a coherent state, $\left|\zeta^{ \pm}\right\rangle$, within one of the, $(x, y)=(0,0)$, SE equilibrium points at any $\epsilon$ value. Then, the probability that any observation results with the system being at its ground state is given by the following expression: 
Fig. 2 Probability to observe a coherent state $\left|\zeta^{ \pm}(0,0)\right\rangle$, in the ground state. $\mathbf{a}$ and $\mathbf{b}$, are related to minima of CES
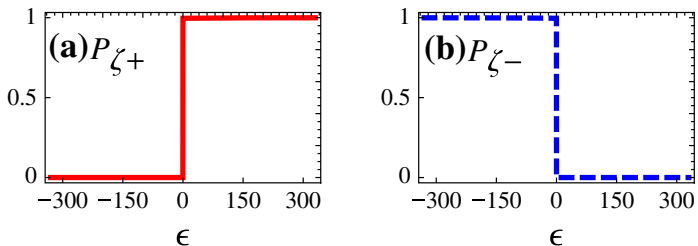

$P_{\zeta^{ \pm}}=\left|\left\langle\zeta^{ \pm}(0,0) \mid \Gamma_{g}(\epsilon)\right\rangle\right|^{2}$

From Eq. (5) we find $\left|\zeta^{ \pm}(0,0)\right\rangle \rightarrow|J, \mp J\rangle$. Thus, from Eq. (4), we conclude that this probability goes through a quantum phase transition at the parameter $\epsilon$ origin, as it is numerically evaluated and plotted in Fig. 2. Reciprocally, this is also a measure of the overlap among the system ground state and the coherent states at these critical points. It is important to note that the ground state overlaps almost completely with a coherent state corresponding to a critical absolute minima. From the qubit behavior standpoint, there is also a sudden inversion, from a constant value true: $(a, b)=(1,0)$, to a false: $(0,1)$, sudden catastrophe, at the critical point parameter $\epsilon=0$ value. Another example of the qubit behavior is given at the separatrix point, $\epsilon=-\epsilon_{x}$. There, the absolute maximum, $(0,0)$, corresponding to the CES $E^{+}$, bifurcates into maxima, $(0,0)$ and saddle points $\left( \pm x_{\mathrm{c}}, 0\right)$, as it is described in Table 1 . On this, initial, coalescence point, the qubit associated with these absolute maximum is $(a, b)=(1,0)$, and as $\epsilon \rightarrow 0$, the ported qubit obeys a linear dependence along this critical point: $\left|a\left(x_{\mathrm{c}}\right)\right|^{2}=-\frac{1}{2 \epsilon_{x}} \epsilon+\frac{1}{2}$, going to $\left|a\left(x_{\mathrm{c}}\right)\right|^{2}=\frac{1}{2}$.

\section{Quantum tunneling: Magnetization and fidelity}

The magnetic properties of the $J=10, \mathrm{Fe}_{8} \mathrm{SMM}$ are well described by the spin Hamiltonian Eq. (1), and we adopted the parameter values related to those given in [5]: $D=\frac{-110.96}{2 J(2 J-1)}(\mathrm{K}), E=0.16|D|$, and $\frac{\epsilon}{4 J}=\mathrm{g}_{e} \mu_{B} \mu_{0} H_{z}$,
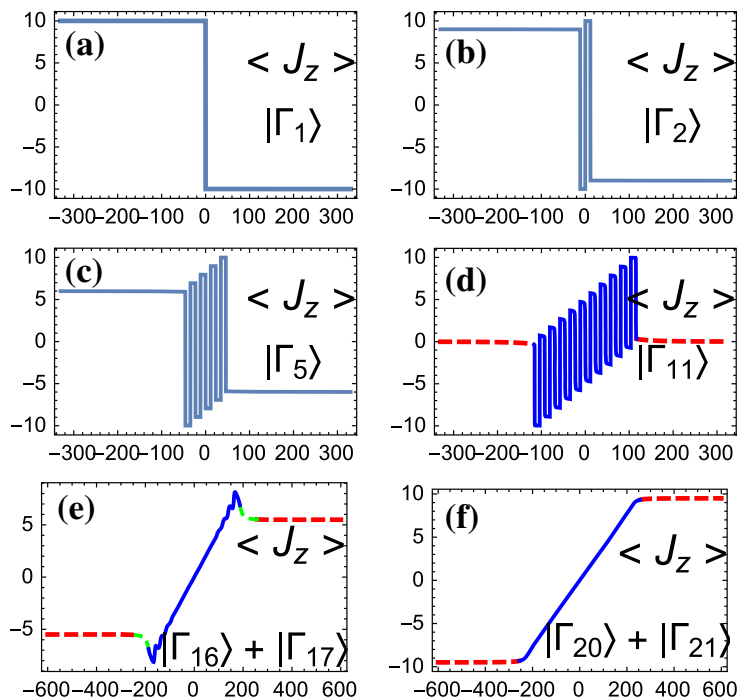

$\epsilon$

Fig. 3 Expectation value $\left\langle J_{z}\right\rangle$ vs. $\epsilon$, evaluated for some eigenstates $\left|\Gamma_{n}\right\rangle$, for $n$ values indicated from (a) to (f). Magnetization of the system, as well as expectation value $\left\langle J_{z}\right\rangle$, evolves as control parameter $\epsilon$ changes adiabatically, going through a quantum phase transition when the system crosses the separatrix, as it is indicated by sudden slope changes, or by dashed line with a different qualitative character. Outside the separatrix the expectation values from (a) to (f) are close to the Dicke numbers, $m=10,9, \ldots,-10$; from (d) to (f) this value is indicated by a dashed line. Within the pairwise avoided level crossing region, expectation value for the corresponding superposition of eigenstates goes through quantitative changes as parameter $\epsilon$ crosses its critical values, as indicated by distinct qualitative dashed line into those graphs 


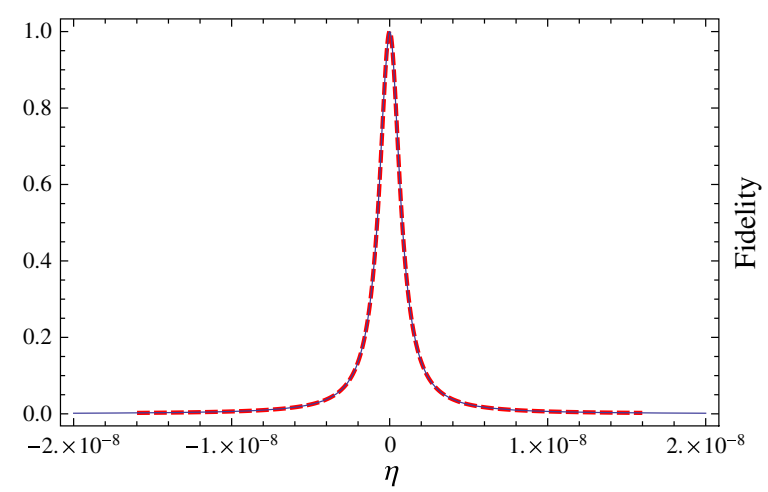

Fig. 4 Fidelity of the ground state at the critical point $\epsilon=0: \mid\left\langle\Gamma_{g}(-\eta)\left|\Gamma_{g}(+\eta\rangle\right|^{2}\right.$, as a function of $\eta$, is plotted (continuous). The Lorentzian curve, $\frac{\delta^{2}}{\delta^{2}+\eta^{2}}$, of the same height and width: $\delta=7.9267 \times 10^{-10}$, is plotted for comparison (dotted). For the first excited state, Fidelity at this critical point follows this same curve; however, the third excited level falls following a much more broader Lorentzian, with $\delta=1.7840 \times 10^{-7}$

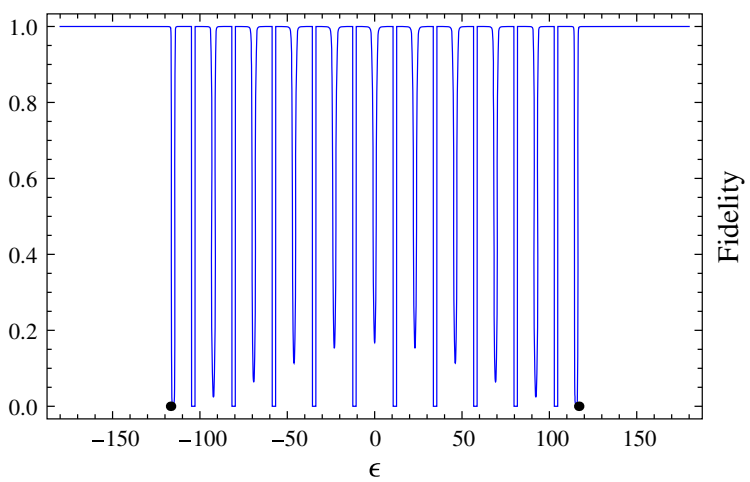

Fig. 5 Fidelity $\mathcal{F}(\epsilon)=\left|\left\langle\Gamma_{11}(\epsilon-\eta) \mid \Gamma_{11}(\epsilon-\eta)\right\rangle\right|^{2}$, for the 11 th excited state at a fixed $\eta=1$ distance. A sudden fall of $\mathcal{F}$, reveals tunneling with both: the above and below closest energy levels. This fall is greater as a nearer avoided energy level crossings takes place. Two points, near to the horizontal axis at $\epsilon= \pm 116.78$ values, are plotted, where the $\Lambda_{11}(\epsilon)$ eigenvalue crosses the $E^{ \pm}(0,0)$ CES

$\mu_{0}$ is the Bohr magneton, $H_{z}$ is the magnetic field $(T)$. The magnetic moment is $\left\langle\mu_{z}\right\rangle=-\mu_{B} g\left\langle J_{z}\right\rangle$. Magnetization of the system, as well as expectation value $\left\langle J_{z}\right\rangle$, evolves as control parameter changes adiabatically, going through a quantum phase transition when the system crosses the separatrix. This sudden phase behavior changes are numerically evaluated and plotted in Fig. 3, for some energy eigenstates as an example. There, one observes a stability behavior for the expectation value $\left\langle J_{z}\right\rangle$ at the outside region of the separatrix and sudden changes at the inner, avoided energy level anticrossings region, due to tunneling. This stability behavior is also observed for the ground, and excited states, quantum Fidelity $[29,30]$. Given any state of the system, $\left|\Psi_{s}(\epsilon)\right\rangle$, its Fidelity at any point in the parameter space is measured in terms of the distance $\eta: \mathcal{F} \equiv\left|\left\langle\Psi_{s}(\epsilon-\eta) \mid \Psi_{s}(\epsilon+\eta)\right\rangle\right|^{2}$. For the ground state, a quantum phase transition occurs at the critical parameter, $\epsilon=0$, as it is revealed by the sudden fall in its Fidelity, shown at Fig. 4. The avoided level crossing parameter region also corresponds to sudden falls for the Fidelity of those excited states within this region, revealing tunneling between almost degenerated states. This is shown for one of those states at Fig. 5.

\section{Conclusions}

In the present work we study a model SMM Hamiltonian. Quantum and semiclassical phase transition behavior are completely organized by the separatrix of the system. Catastrophe Theory and Coherent State formalisms play 
the role of a powerful combined tool to tackle a variety of problems in the study of quantum information. Coherent qubit states are introduced by means of the coherent state representation of the system states. Coherent states represent very closely the ground state of the system and demonstrate their importance when they are evaluated at the semiclassic critical points of the ES to study the singular character, due to tunneling, of Fidelity at phase transition points. This deserves further investigation, as it is suggested by its Lorentzian decay at QPT points.

Acknowledgments ELM is a tutorial fellow Carlos Graef Fernández from Facultad de Ciencias, UNAM. We acknowledge the support from UNAM-DGAPA PAPITT-IN117811 and IN117911 projects.

\section{References}

1. Castaños, O., López-Peña, R., Hirsch, J.G., López-Moreno, E.: Phase transitions and accidental degeneracy in nonlinear spin systems. Phys. Rev. B 72, 012406 (2005)

2. Vidal, J., Palacios, G., Mossen, R.: Entanglement in a second-order quantum phase transition. Phys. Rev. A 69, 022107 (2004)

3. Vidal, J., Mossen, R., Dukelsky, J.: Entanglement in a first-order quantum phase transition. Phys. Rev. A 69, 054101 (2004)

4. Roscilde, T., Verrucchi, P., Fubini, A., Hass, S., Tognetti, V.: Studying quantum spin systems through entanglement estimators. Phys. Rev. Lett. 93, 167203 (2004)

5. Gatteschi, D., Sessoli, R., Villain, J.: Molecular Nanomagnets. Oxford University Press, New York (2006)

6. Barra, A.L., Gatteschi, D.L., Sessoli: high-frequency EPR spectra of $\left[\mathrm{Fe}_{8} \mathrm{O}_{2}(\mathrm{OH})_{12}(\mathrm{tacn})_{6}\right] \mathrm{Br}_{8}$ : a critical appraisal of the barrier for the reorientation of the magnetization in single-molecule magnets. R. Chem. Eur. J. 6, 1608-1614 (2000)

7. Wernsdorfer, W., Sessoli, R., Caneshi, A., Gatteschi, D.: Landau-Zener method to study quantum phase interference of Fe 8 molecular nanomagnets (invited). J. Appl. Phys. 87, 548-5489 (2000)

8. Wernsdorfer, W.: Quantum dynamics in molecular nanomagnets. C. R. Chimie 11, 1086-1109 (2008)

9. Takahashi, S., Tupitsyn, I.S., van Tol, J., Beedle, C.C., Hendrickson, D.N., Stamp, P.C.E.: Decoherence in crystals of quantum molecular magnets. Nature 476, 76-79 (2011)

10. Takahashi, S., van Tol, J., Beedle, C.C., Hendrickson, D.N., Brunel, L.-C., Sherwin, M.S.: Coherent manipulation and decoherence of $S=10$ single-molecule magnets. Phys. Rev. Lett. 102, 087603 (2009)

11. Sachdev, S.: Quantum Phase Transitions. Cambridge University Press, Cambridge (1999)

12. Caprio, M.A., Cejnar, P., Iachello, F.: Excited state quantum phase transitions in many-body systems. Ann. Phys. 323, 1106-1135 (2008)

13. López-Moreno, E., Castaños, O.: Shapes and stability within the interacting boson model: dynamical symmetries. Phys. Rev. C 54, 2374 (1996)

14. Lipkin, H.J., Meshkov, N., Glick, A.J.: Validity of many-body approximation methods for a solvable model: (I). Exact solutions and perturbation theory. Nucl. Phys. 62, 188, 199, 211 (1965)

15. Ginocchio, J.N., Kirson, M.W.: Relationship between the Bohr collective Hamiltonian and the interacting Boson model. Phys. Rev. Lett. 44, 1744 (1980)

16. Dieperink, A.E.L., Scholten, O., Iachello, F.: Classical limit of the interacting Boson model. ibid. 44, 1747 (1980)

17. Castaños, O., López-Peña, R., Hirsch, J.G., López-Moreno, E.: Classical and quantum phase transitions in the Lipkin-MeshkovGlick model. Phys. Rev. B 74, 104118 (2006)

18. Ribeiro, P., Vidal, J., Mosseri, R.: Thermodynamical limit of the Lipkin-Meshkov-Glick model. Phys. Rev. Lett. 99, 050402 (2007)

19. Ribeiro, P., Vidal, J., Mosseri, R.: Exact spectrum of the Lipkin-Meshkov-Glick model in the thermodynamic limit and finite-size corrections. Phys. Rev. E 78, 021106 (2008)

20. Orús, R., Dusuel, S., Vidal, J.: Equivalence of critical scaling laws for many-body entanglement in the Lipkin-Meshkov-Glick Model. Phys. Rev. Lett. 101, 025701 (2008)

21. Kwok, H.-M., Ning, W.-Q., Gu, S.-J., Lin, H.-Q.: Quantum criticality of the Lipkin-Meshkov-Glick model in terms of fidelity susceptibility. Phys. Rev. E 78, 032103 (2008)

22. Rams, M.M., Damski, B.: Quantum Fidelity in the thermodynamic limit. Phys. Rev. Lett. 106, 055701 (2011)

23. Arecchi, F.T., Courtens, E., Gilmore, R., Thomas, H.: Atomic coherent states in quantum optics. Phys. Rev. A 6, 2211 (1972)

24. López-Moreno, E., Grether, M., Velázquez, V.: Energy level structure and quantum phase transitions of spin systems with nonaxially symmetric Hamiltonians. J. Phys. A Math. Theory 44, 475301 (2011)

25. Glauber, R.J.: The quantum theory of optical coherence. Phys. Rev. 130, 2529 (1963). ibid. Coherent and Incoherent States of the Radiation Field. 131, 2766 (1963)

26. Gilmore, R.: On the properties of coherent States. Rev. Mex. Fis. 23, 143-187 (1974)

27. Bloch, F.: Nuclear Induction. Phys. Rev. 70, 460 (1946)

28. Nielsen, M.A., Chuang, I.I.: Quantum computation and quantum information, 10th Anniversary Edition, vol. 174. Cambridge University Press, Cambridge (2010)

29. Jozsa, R.: Fidelity for mixed quantum states. J. Mod. Optics 41, 2315-2323 (1994)

30. Li-Jun, T., Chang-Quing, Z., Hong-Biao, Z., Li-Guo, Q.: Fidelity susceptibility and geometric phase. Chin. Phys. B 20(4), 040302 (2011) 